\title{
Pathological diagnosis of peritoneal loose body: a case report
}

\author{
Rajbhandari $\mathrm{M}^{1}$, Karmacharya $\mathrm{A}^{2}$, Shrestha $\mathrm{S}^{1}$ \\ ${ }^{1}$ Department of Pathology, Dhulikhel Hospital, Nepal \\ ${ }^{2}$ Department of Surgery, Dhulikhel Hopsital, Nepal
}

\section{Keywords:}

Peritoneum;

Loose bodies;

Appendices epiploicae

\begin{abstract}
Peritoneal loose bodies are usually incidental findings at laparotomy. Their sizes range from that of a pea to giant loose bodies. We report a case of giant loose peritoneal body measuring $5 \mathrm{x} 4 \mathrm{~cm}$ found incidentally in a 67 year old man. Mobile pelvic masses are extremely rare findings. They are usually located in the pelvic cavity due to the fact that they gravitate to the most dependent part of the pelvic cavity. Usually these peritoneal loose bodies are left untreated until complications arise.
\end{abstract}

\section{INTRODUCTION}

Peritoneal bodies are rare lesions which are usually asymptomatic. They are occasionally found at laparotomy and are usually small sized, like peas $(1-2 \mathrm{~cm})$. The loose bodies measuring more than $5 \mathrm{cms}$ are even rarer and termed as Giant Loose Bodies. ${ }^{1,2}$ The main etiologies of loose bodies are appendices epiploicae and are considered to be formed by collection of peritoneal serum albumin around infarcted appendices epiploica. ${ }^{3,4}$ We report a case of a giant loose body with focus on the formation of such loose bodies.

\section{CASE REPORT}

A sixty seven-years old farmer came to Dhulikhel Hospital with complaints of pain and weakness of right limb for two months. On physical examination the general condition was good with a pulse rate of $80 / \mathrm{min}$ and blood pressure of 90mm Hg. On examination of lower limb motor function was within normal limits and sensory was intact. Per

\section{Correspondence:}

Dr. Merina Rajbhandari, $M D$

Department of Pathology, Dhulikhel Hospital, Dhulikhel, Nepal.

Email: rajbhandarimerina@hotmail.com abdominal examination revealed mass in the suprapubic region measuring $5 \times 4 \mathrm{cms}$, firm, non-tender with horizontal mobility and limited vertical mobility.

Ultrasound showed a presence of hypoechoeic mass in the right lower abdomen adjacent to urinary bladder with central echogenic focus and was advised for contrast enhanced computed tomography (CECT) for further evaluation. The CT scan showed a well-defined mass enhancing soft tissue density mass lesion with central hyper dense foci in pelvic cavity abutting posterior wall of urinary bladder suggesting of bowel mass. The diagnostic laparoscopy was performed which revealed a presence of a single oval shaped freely mobile mass of size of roughly hens egg completely free in the peritoneal cavity. All the other organs were normal.

The mass was sent to the Pathology department for the diagnosis. Macroscopically, the loose body was oval shaped, measuring $5 \mathrm{cms}$ in length and $4 \mathrm{cms}$ in width. The external surface was smooth, dirty white in color and hard in consistency (fig.1A). Cut surface revealed central core which was yellowish area with white laminated area at the periphery (fig.1B \&C).

Histologically, this body consisted of laminated strands 


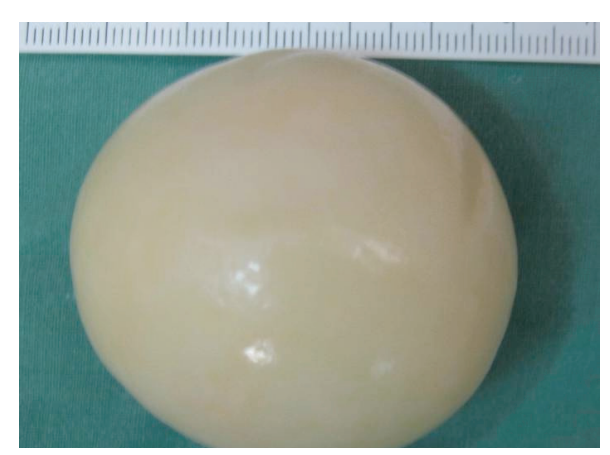

Figure 1A: Gross picture of Peritoneal loose body measuring $5 \times 4 \mathrm{cms}$.

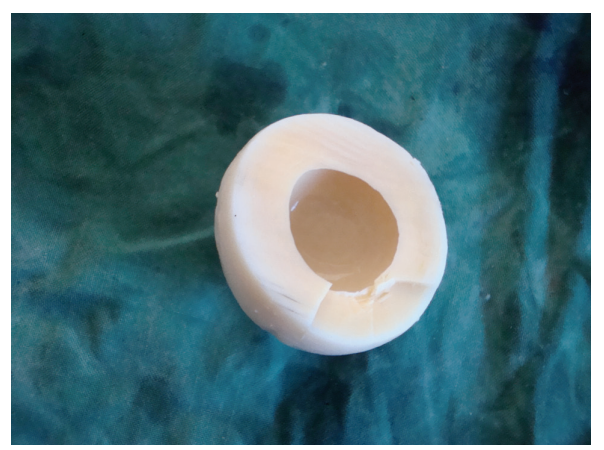

Figure 1C: The outer region after removal of central yellowish region.

of hyalinized material at the periphery with central area showing necrotic fat with areas of calcification (fig. 2 and fig.3).

\section{DISCUSSION}

It was Harrigan who first described free-lying appendix epiploicae. ${ }^{5}$ Appendices epiploicae containing fat tissue are present along the entire length of colon. ${ }^{6}$ Virchow in 1863 proposed the theory of formation of loose peritoneal body. ${ }^{6}$ According to him, increase in fat content of appendices epiploicae leads to gradual and progressive obliteration or obstruction of blood vessels of pedicle. The torsion, strangulation, and necrosis of epiploic appendages may occur due to inflammation caused by intra-abdominal adhesion, volvulus, intestinal obstruction, or intestinal perforation. The pedicle atrophies and gets detached from the colon due to ischemia and thus forming the loose body. ${ }^{7,8,9}$

These loose bodies are usually found accidentally found during surgery or autopsy. They have similar features with white or gray with a soft and light margin and have rubbery consistency. In general, it has a diameter of about 0.5 to 2.5 $\mathrm{cm}$ and is freely separated from the inside of the abdominal cavity. The separated appendices epiploicae undergoes gradual saponification and calcification. The peritoneal fluid rich in protein, mainly albumin accumulates around it and causing gradual increase in the size of the loose body.

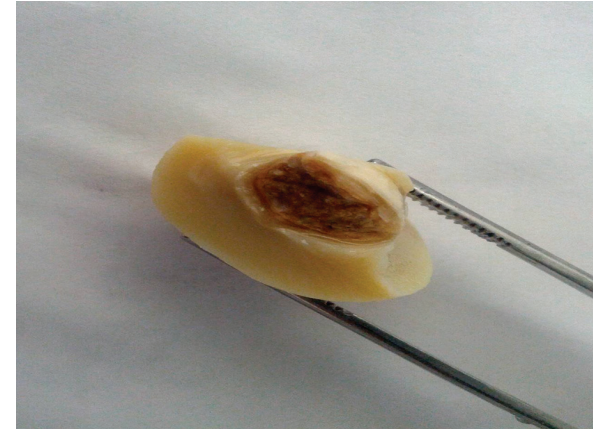

Figure $1 B$ : Cut section showing central yellowish calcified area surrounded by concentric laminated whitish region.

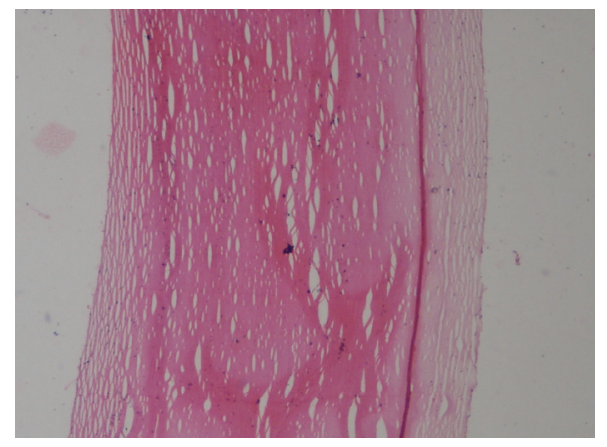

Figure 2: Microscopic picture showing outer laminated hyalinized area (HE Stain, X100).

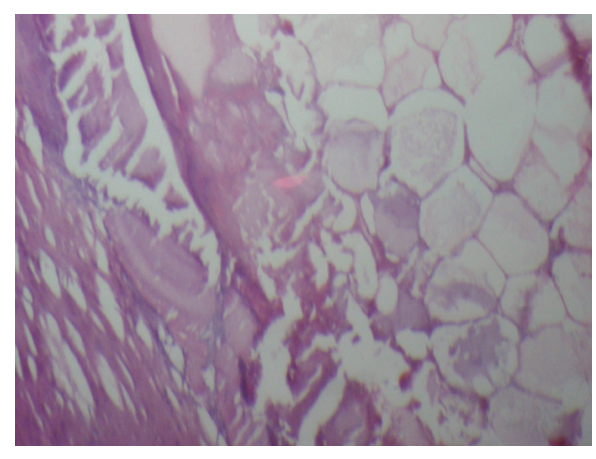

Figure 3: Microscpoic picture with central fat necrosis with calcification (HE Stain, X400).

Except for a loose body that has undergone normal calcification, no particular pathological importance is found, and no pathology or frequency data are available. Peritoneal loose bodies may present with no symptoms like as in our case or may present with various symptoms due to compression effects like abdominal pain, constipation , acute retention of urine and intestinal obstruction. ${ }^{10}$

\section{CONCLUSION}

A giant loose body with a size of more than $5 \mathrm{cms}$ in diameter is very difficult to diagnose before surgery and is rarely diagnosed and reported in clinical practices. Until now, no such case has been reported in Nepal. However, the giant loose body has to be separated from other causes like 
tumors, ovarian lesions and other causes.

\section{REFERENCES}

1. Ghosh P, Strong C, Naugler W, Haghighi P, Carethers JM. Peritoneal mice implicated in intestinal obstruction: report of a case and review of the literature. J Clin Gastroenterol 2006;40:427-30.

2. Shepherd JA. Peritoneal loose body causing acute retention of urine. Br J Surg 1951;39:185-7.

3. Ross JA, McQueen A. Peritoneal loose bodies. BrJ Surg 1947;35:3137.

4. Hedawoo J.B, Wagh A. Giant peritoneal loose body in a patient with hemorrhoids. Tropical Gastroenterology 2010;31:132-3.

5. Harrigan AH. Torsion and inflammation of the appendix epiploicae. Ann Surg. 1917;66:467-78.
6. Borg SA, Whitehouse GH, Griffiths GJ. A mobile calcified amputed appendix epiploica. Am J Rentogenol. 1976;127:349-50.

7. Takada A, Moriya Y, Muramatsu Y, Sagae T. A case of giant peritoneal loose bodies mimicking calcified leiomyoma originating from the rectum. Jpn J Clin Oncol. 1998;28:441-2.

8. Desai Helicobacter pylori, Tripodi J, Gold BM, Burakoff R. Infarction of an epiploic appendage. Review of the Literature. J Clin Gastroenterol 1993;16:323-5.

9. Patterson DC. Appendices epiploicae and their surgical significance with report of three cases. N Eng J Med 1933;209:1255-9.

10. Gayer G, Petrovitch I. CT diagnosis of a large peritoneal loose body: a case report and review of the literature. Br J Radiol 2011;84:83-85. 